\title{
Cholinesterase variants: rapid characterisation by PCR/SSCP and evidence for molecular homogeneity
}

Thomas Höhler, Matthias Hundt, Christian Rittner, Peter M Schneider, Karl-H Meyer zum Büschenfelde

\begin{abstract}
We have applied the technique of PCRSSCP (polymerase chain reaction - single stranded conformation polymorphism) to characterise the molecular basis of cholinesterase deficiency and variants in a Jordanian family. PCR-SSCP proved to be a quick and sensitive method of screening cholinesterase variants in a clinical setting. An AG insertion at position 351 was found to cause a silent allele, for which the parents were heterozygous and three children homozygous. In addition, the father and two sons were heterozygous for an $A$ to $G$ transition at position 209 , known to cause the dibucaine resistant variant. No linkage to the $K$ variant was found, which has been reported previously in white populations. These findings suggest considerable homogeneity in the molecular basis of CHE variants between different ethnic groups.
\end{abstract}

( $($ Med Genet 1995;32:109-112)

Serum cholinesterase (CHE, butyrylcholinesterase) is a cholinester hydrolysing enzyme that is synthesised in the liver. Its activity is measured in routine laboratory tests to assess liver function and monitor intoxication with organophosphates. The physiological function of the enzyme is unknown, but patients with acholinesterasaemia or certain cholinesterase variants are known to have prolonged duration of inhibition of neuromuscular transmission induced by succinylcholine. ${ }^{1}$

The molecular bases of some structural vari-

I Med Klinik

und Poliklinik,

Johannes Gutenberg-

Universität Mainz,

Langenbeckstrasse 1,

55101 Mainz, Germany

T Höhler

$M$ Hundt

K-H Meyer zum

Büschenfelde

Institute for Legal

Medicine, Johannes

Gutenberg University,

Mainz, Germany

C Rittner

P M Schneider

Correspondence to: Professor Meyer zum Büschenfelde.

Received 28 June 1994

Revised version accepted for Revised version accepted for
publication 7 October 1994
The entire coding region of the gene can be amplified with only a few PCR primers using genomic DNA. The consequences of mutations are easily assessed by measuring the $\mathrm{CHE}$ activity with routine laboratory tests.

To characterise the $\mathrm{CHE}$ variant of a patient with acholinesteraemia and her family, we developed a strategy based on PCR amplification and genomic DNA sequence analysis. We selected four pairs of primers from the published CHE genomic sequence 5 covering the three exons that code for the mature protein. The amplified DNA was subjected to single stranded conformation polymorphism (SSCP) analysis to detect possible mutations. Finally, direct PCR sequencing of the PCR product exhibiting a variation in the SSCP pattern was used to define the type of mutation.

\section{Methods}

PATIENTS

A 20 year old woman from Jordan was admitted to our hospital because of acute hepatitis B. A very low $\mathrm{CHE}$ activity was found in her serum. After informed consent, blood was also taken from her parents, her sister, and her three brothers. DNA was isolated according to standard methods. Serum cholinesterase phenotypes were determined from the rate of benzoylcholine hydrolysis, the dibucaine inhibition number, ${ }^{1}$ and the fluoride inhibition number. ${ }^{6}$ Inhibition numbers could not be determined in one brother, one sister, and the patient herself because of the very low CHE activity.

PCR AMPLIFICATION

Four pairs of oligonucleotide primers were chosen according to the published CHE genomic sequence from flanking intron sequences ${ }^{5}$ as shown in the table. The downstream primer of each primer pair was biotinylated. With these primers all three exons coding for the mature CHE protein can be amplified. PCR was performed in a thermocycler (9600, Perkin Elmer Corp, Norwalk, CT) with 100 ng of genomic DNA, $25 \mathrm{pmol}$ of each primer, $200 \mu \mathrm{mol}$ total dNTP, and $2 \mathrm{U}$ Taq polymerase (Boehringer, Mannheim) using a "touchdown" protocol. The first six cycles were carried out at decreasing annealing temperatures in $1^{\circ} \mathrm{C}$ steps for one cycle each from $65^{\circ}$ to $60^{\circ} \mathrm{C}$, followed by 25 cycles using the following conditions: 30 seconds $94^{\circ} \mathrm{C}$; one minute $59^{\circ} \mathrm{C}$; one minute $72^{\circ} \mathrm{C}$. For primers $45 / 43$ and $55 / 53$ a different
Studies of CHE gene alterations are a valuable model for other gene mutations in man. frameshift mutation at position 351 (GGT $\rightarrow$ GGAG) has been identified as the basis of one type of silent mutation. ${ }^{3}$ In another family inactivation of the cholinesterase gene was caused by an Alu insertion in exon 2. 
Primers used in the study

\begin{tabular}{lllc}
\hline Primer & Sequence $\left(5^{\prime}-3^{\prime}\right)$ & Position & Size $(b p)$ \\
\hline 15 & TGGTTTCTTTTGCTCTGCAT & $-42--23$ & 465 \\
13 & TACAATAACTCTTTCAACCCG & $423-403$ & 1088 \\
25 & CATGTTTATGATGGCAAG & $376-393$ \\
33 & CAGAGAACAATGACAAAA & Intron 2* & 237 \\
45 & CAATTTTACTATAATGTCTC & Intron 2* & \\
43 & TCTATTAAATAACCAAACAC & Intron 3 & 189 \\
53 & TGATTAATACAACTTATTCC & Intron 3* & \\
$55 / 5$ & GAAAATATGTTCTATAAAGGG & $3^{\prime}$ untranslated region & \\
seq5 & TTTCAGTTAATGAAACAGAT & Intron 3* & $172-192$ \\
\hline
\end{tabular}

*Intron primers are all located approximately $30 \mathrm{bp}$ within the intron.

programme without touchdown was carried out, which used an annealing temperature of $50^{\circ} \mathrm{C}$ for all 30 cycles.

\section{SSCP ANALYSIS}

The PCR product from the amplification of primers $25 / 33$ was purified by adsorbtion to glass milk (Geneclean; BIO 101, La Jolla, CA) and digested with $H p h I$ to obtain fragments smaller than $400 \mathrm{bp}$. The digested fragments, as well as the other PCR products, were denatured with formamide by heating at $80^{\circ} \mathrm{C}$ for three minutes and kept on ice until loading. The mixture was separated in a $0.75 \mathrm{~mm}$ thick MDE gel in $0.6 \times$ TBE buffer (AT Biochem, Malvern, PA), polymerised on Gel-Bond PAG film
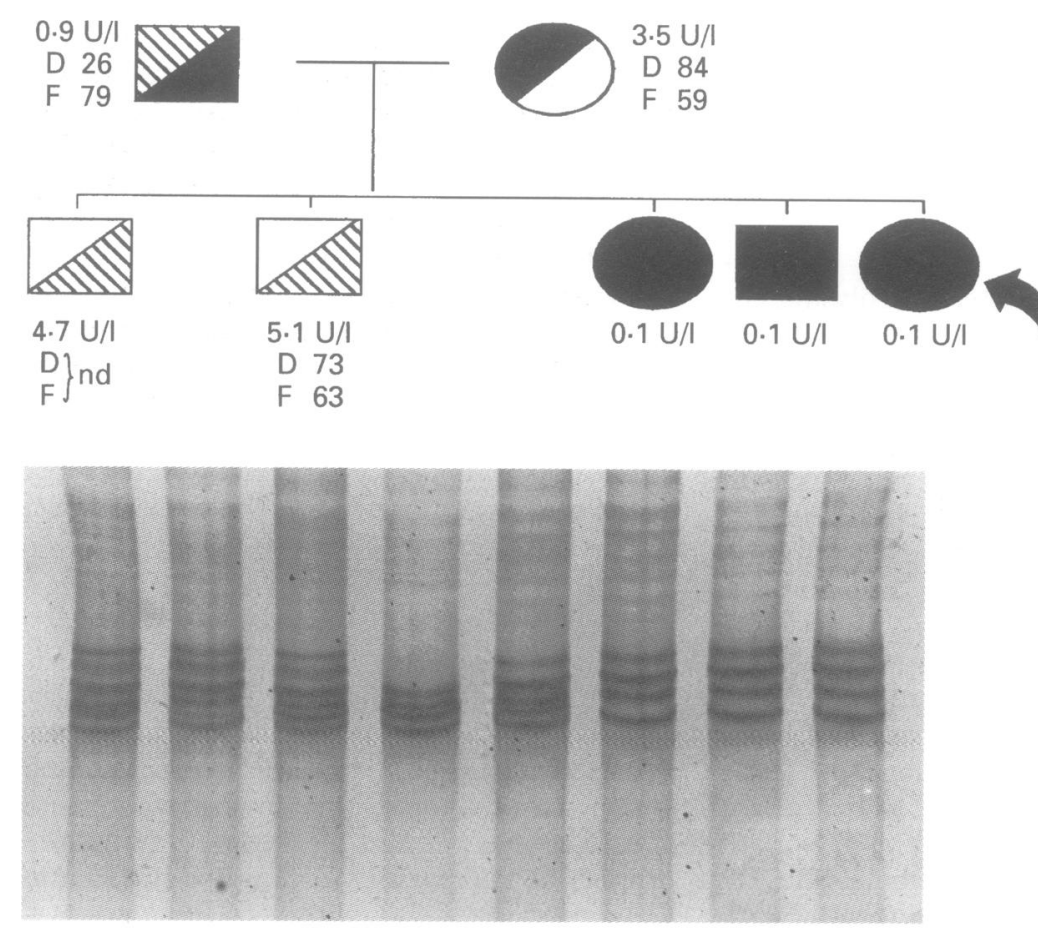

C

Figure 1 Family pedigree illustrating inheritance of $C H E$ variants. The patient is indicated by an arrow. Serum cholinesterase activities (normal 3.0-7.5 kU/l) as well as dibucaine numbers ' (normal 80-88, atypical heterozygous 60-68, atypical homozygous 12-25) and fluoride numbers" (normal 40-60, atypical heterozygous 50-66, atypical homozygous 74-84) are shown. Inihibition numbers could not be determined in one brother, one sister, and the patient herself because of the very low CHE activity. Inhibition numbers were not determined (nd) in the person on the bottom left side of the pedigree. Silent alleles are represented by solid symbols, open symbols denote normal alleles, and hatched symbols indicate the dibucaine resistant allele. The photo shows the SSCP analysis of fragment 15-13. Four characteristic patterns can be distinguished $(C=$ control).
(Pharmacia LKB, Freiburg) at room temperature. The gel was electrophoresed at $400 \mathrm{~V}$ for 10 to 18 hours. ${ }^{89}$ The DNA was visualised by silver staining, as previously described with minor modifications. ${ }^{10}$

GENOMIC DNA SEQUENCING

For direct sequencing of PCR amplified products, single stranded DNA was obtained using streptavidin conjugated magnetic beads (Dynal, Norway). The sequencing reaction was carried out on single stranded DNA using the T7 Sequencing Kit (Pharmacia LKB, Freiburg) and $\alpha-\left[{ }^{35}\right.$ S $]$ dATP (Du Pont NEN, Dreiech).

\section{Results}

Results of CHE standard activity assay and inhibition tests are shown in fig 1 indicating the codominant inheritance of the $\mathrm{CHE}$ alleles. The very low CHE activity in our patient (arrow), one brother, and one sister is characteristic of persons who are homozygous for silent alleles. The very low dibucaine inhibition number and the reduced CHE activity of the father suggest that he is either homozygous for the dibucaine resistant variant or heterozygous for this variant and a silent allele. Consequently the two brothers with normal CHE activity have to be heterozygous for the dibucaine resistant variant as suggested by the dibucaine inhibition number.

SSCP analysis of PCR fragments 55-53, 45-43, and of the $H p h \mathrm{I}$ digested fragments 25-33 (data not shown) did not show any differences between the DNA of the control subject and the family members. Characteristic SSC polymorphisms were obtained using primers 15 and 13, which amplify the $5^{\prime}$ end of the protein coding region from nucleotide -42 to 423 (fig 1). SSCP patterns reflect exactly the inheritance of the CHE alleles. All four bands in the three children with very low CHE activity differ from the control, suggesting that both their CHE alleles are different from the wild type. The band pattern of the mother appears to be a combination of the control and the deficient children presumably caused by heterozygosity for the wild type and a mutated allele. A similarly composed pattern can be observed for the two brothers with normal $\mathrm{CHE}$ activity. The patient and the control samples show four distinct bands instead of the expected 
A

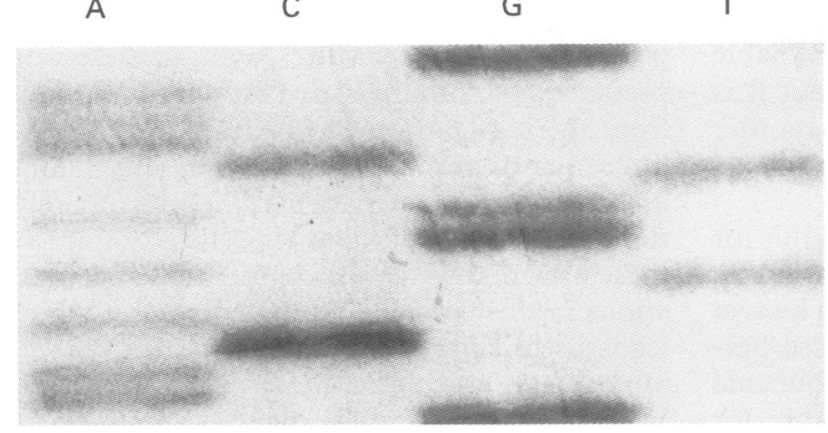

Figure 2 DNA sequence of CHE exon 2 (father, coding strand) obtained by PCR amplification with primers $15 / 13$ and sequencing with primer 15 . The $A$ to $G$ mutation causes dibucaine resistance.

Sequencing of fragment seq5/13, which covers $252 \mathrm{bp}$ at the $3^{\prime}$ end of fragment $15 / 13$, with sequencing primer seq5 showed a frameshift mutation (GGT $\rightarrow$ GGAG) at position 351 , resulting in the appearance of a stop codon at nucleotide 384 . Both parents were found to be heterozygous for this mutation. Two sisters and one brother with very low $\mathrm{CHE}$ activity were homozygous for the insertion (fig 3). This mutation is known to cause a silent variant. ${ }^{3}$

A guanine to adenine polymorphism at nucleotide position 1615 has been found to cause the $\mathrm{K}$ variant, which is characterised by a reduction of about one third in cholinesterase activity. ${ }^{41112}$ This polymorphism appears to be in close linkage to the mutation at position 209, which causes the dibucaine resistant variant. SSCP analysis of fragment 55/53, which covers the $\mathrm{K}$ variant coding region, did not show any difference between family members and the control. We therefore decided to sequence this fragment with primers $55 / 5$ and 53 using primer $55 / 5$ as sequencing primer. Sequencing results showed guanine at position 1615 in all family members (data not shown). sons with normal CHE activity showed an additional band at $274 \mathrm{bp}$ (data not shown) on a polyacrylamide gel. Therefore this fragment was selected for detailed DNA sequence analysis.

Genomic DNA sequence analysis of this fragment using PCR primer 15 as sequencing primer showed an adenine to guanine mutation a nucleotide 209 , causing a change of aspartate (GAT) to glycine (GGT) at position 70 . The father and the two sons with the normal CHE were found to be heterozygous for this mutation (fig 2). This mutation is known to cause the dibucaine resistant variant ${ }^{8}$ and the loss of a $S a u 3 A$ restriction site (GATC $\rightarrow$ GGTC).

A

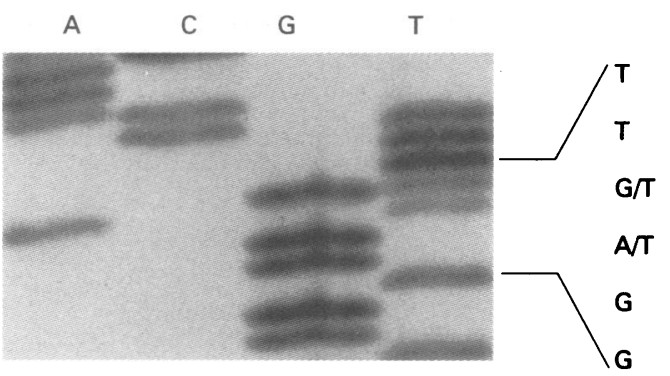

B

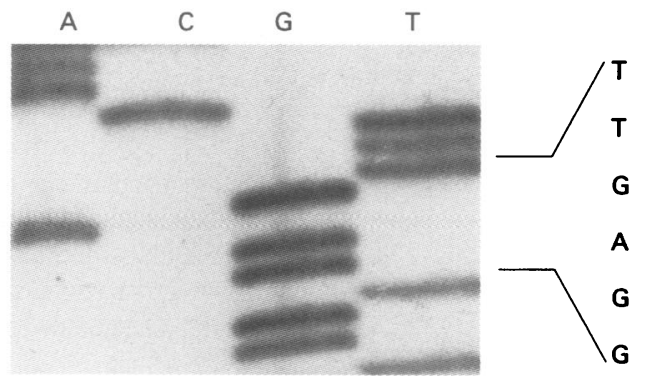

Figure 3 Direct sequencing (coding strand) with primer seq5 of DNA amplified with primers seq5/13. (A) Mother heterozygous for the $G A$ insertion at position 351 . (B) Patient (arrowed in fig 1), homozygous for the insertion.

\section{Discussion}

We have applied PCR-SSCP analysis to characterise the molecular defect in a family with acholinesterasaemia. This technique proved to be a rapid and powerful approach to screen the CHE gene for mutations. The mutations detected, the $G$ to $A$ transition at nucleotide 209 as well as the AG insertion at nucleotide 351 , are within fragment $15-13$. They both caused characteristic SSCP resulting in four different band patterns for the entire family (fig 1). The SSCP technique is most sensitive when examining DNA fragments of 100 to $300 \mathrm{bp}$, showing mobility shifts of at least one strand in 70 to $99 \%$ of mutations. ${ }^{8}$ The sensitivity of the method decreases with the size of the PCR product and is less than $50 \%$ when the fragment is $>400 \mathrm{bp}$. To overcome this size limitation we digested fragment $25-33$ (1088 bp) with $H$ phI before SSCP analysis.

A number of $\mathrm{CHE}$ variants have been described and their molecular bases have been characterised. ${ }^{2-4}$ Our interest in this family arose from two observations. Cholinesterase activities in the parents did not seem to fit well with current knowledge of $\mathrm{CHE}$ variants. In addition, family members were Jordanian Arabs and little is known about the molecular basis for a silent allele as three children were expected to be homozygous for a silent allele (fig 1). Despite the suspected heterozygosity the mother had a normal CHE activity. The dibucaine inhibition number of the father suggested that he was either homozygous for the dibucaine resistant variant or heterozygous for dibucaine resistance and a silent variant. In either case two of the sons had to be heterozygous for the dibucaine resistant variant, despite their high CHE activity, because they were not carriers of a silent allele.

The mother and the father were found to be heterozygous and three children homozygous for a GGT to GGAG frameshift mutation. This mutation leads to a stop codon at nucleotide 
384, generating a truncated protein that lacks the active catalytic site. ${ }^{3}$ No data are available on the frequency of the silent allele, but it is probably very rare. ${ }^{2}$ Its presence in the mother and the father in this family is most likely because they are cousins.

Furthermore the father was heterozygous for a point mutation at nucleotide 209 , known to cause the dibucaine resistant variant. ${ }^{3}$ The two sons with normal CHE activity were also heterozygous for this mutation. The dibucaine resistant variant shows a reduced affinity for binding cholinester substrates such as succinylcholine and a reduced degree of inhibition by dibucaine. ${ }^{13}$ This variant is clinically important because the muscle relaxant succinylcholine is frequently used in anaesthesia. In whites the atypical allele has a gene frequency of $0.017^{14}$ and about 3 in 10000 are homozygous for the atypical variant.

In a North American population the dibucaine resistant variant has been found to be closely linked to a polymorphism at position 1615 resulting from a $G$ to $A$ transition $(\mathrm{GCA} \rightarrow \mathrm{ACA}){ }^{4}$ The frequencies of the polymorphic alleles are 0.875 (GCA) and 0.125 (ACA). ACA codes for the K variant, a quantitative variant with an activity reduced by $33 \%{ }^{412}$ It is believed that the linkage to the $\mathrm{K}$ variant accounts for the fact that atypical serum contains one third less esterase protein than is present in usual serum. ${ }^{411}$ SSCP analysis of fragment 55-53, which contains this polymorphism, did not show any differences between the control and family members. We therefore proceeded to sequence this fragment with primer $55 / 5$. We found GCA coding for alanine in all four alleles of the parents. This finding probably explains the normal $\mathrm{CHE}$ activity in the two sons despite their being heterozygous for the dibucaine resistant variant. In addition, it supports the hypothesis of Bartels et $a l^{4}$ that the $\mathrm{K}$ variant is responsible for the observed reduction in $\mathrm{CHE}$ activity in atypical serum.

As $\mathrm{CHE}$ variants and deficiencies have no influence on health apart from prolonged muscle relaxation after succinylcholine they presumably could evolve without selection pressure to eliminate certain $\mathrm{CHE}$ variants from the population. Most of the work on the molecular basis of CHE variants has been done in North American patients. ${ }^{34}$ Unfortunately, the authors do not state the ethnic origin of these patients. We could identify the same mutations in this family of Jordanian Arabs, which leads us to suppose that there is considerable molecular homogeneity between ethnic groups. With regard to the dibucaine resistant variant our family did not show the linkage to the $K$ variant that has been reported from North America. ${ }^{4}$

1 Kalow W, Gunn DR. The relation between dose of succinylcholine and duration of apnea in man. $\mathcal{F}$ Pharmacol Exp Ther 1957;120:203-14.

2 Muratani K, Hada Yamamoto Y, et al. Inactivation of the cholinesterase gene by Alu insertion: possible mechanism for human gene transposition. Proc Natl Acad Sci USA 1991;88:11315-19.

3 McGuire MC, Nogueira CP, Bartels CF, et al. Identification of the structural mutation responsible for the dibucaineresistant (atypical) variant form of human serum cholinesterase. Proc Natl Acad Sci USA 1989;86:953-7.

4 Bartels CF, Jensen FS, Lockridge O, et al. DNA mutation associated with the human butyrylcholinesterase $\mathrm{K}$-variant and its linkage to the atypical variant mutation and other and its linkage to the atypical variant mutation and other

polymorphic sites. Am f Hum Genet 1992;50:1086-103.
5 Arpagaus M, Kott M, Vatsis KM, Bartels CF, La Du BN, Arpagaus $M$, Kott M, Vatsis KM, Bartels $\mathrm{CF}, \mathrm{La} \mathrm{Du} \mathrm{BN}$,
Lockridge $O$. Structure of the gene for human butyrylcholinesterase. Evidence for a single copy. Biochemistry tyrylcholinesteras

6 Harris $\mathrm{H}$, Whittaker $M$. Differential inhibition of human serum cholinesterase with fluoride: recognition of two new phenotypes. Nature 1961;191:496-8.

7 Don RH, Cox PT, Wainwright BJ, Baker K, Mattick JS. 'Touchdown' PCR to circumvent spurious priming during gene amplification. Nucleic Acids Res 1991;19:4008.

8 Hayashi K. PCR-SSCP: a simple and sensitive method for detection of mutations in genomic DNA. PCR Methods and Applications 1991;1:34-8.

9 Spinardi L, Mazars R, Theillet C. Protocols for an improved detection of point mutations by SSCP. Nucleic Acids Res detection of point

10 Sammons DW, Adams LD, Nishizawe EE. Ultrasensitive silver-based color staining of polypeptides in polysilver-based color staining of polypeptides
acrylamide gels. Electrophoresis $1981 ; 2: 135-41$.

11 Eckerson HW, Oseroff A, Lockridge O, La Du BN. Immunological comparison of the usual and atypical human serum cholinesterase phenotypes. Biochem Genet 1983;21: 93-108

12 Rubinstein $\mathrm{HM}$, Dietz AA, Lubrano T. $\mathrm{E}_{1}{ }^{\mathrm{K}}$, another quantitative variant at cholinesterase locus I. $f$ Med Genet 1978 ; 15:27-9.

13 Kalow W, Davies RO. The activity of various esterase inhibitors towards atypical human serum cholinesterase. Biochem Pharmacol 1958; 1:183-92.

14 Whittaker M. Cholinesterase. Basel: Karger, 1986. 